\title{
Total knee arthroplasty in patients with Ranawat type-Il valgus arthritic knee with a marked coronal femoral bowing deformity: comparison between computer-assisted surgery and intra-articular resection
}

Tsan-Wen Huang ${ }^{1,3^{*}}$, Po-Yao Chuang ${ }^{1}$, Chien-Yin Lee ${ }^{1}$, Shih-Jie Lin ${ }^{1}$, Kuo-Chin Huang ${ }^{1,3}$, Shih-Hsun Shen ${ }^{1}$, Yao-Hung Tsai ${ }^{1,3}$, Mel S. Lee ${ }^{2,3}$ and Robert Wen-Wei Hsu ${ }^{1,3^{*}}$

\begin{abstract}
Background: Proper limb and component alignments as well as soft tissue balance are vital for the longevity and optimal long-term outcomes of total knee arthroplasty (TKA). This procedure is technically demanding in patients with Ranawat type-II valgus arthritic knees with marked coronal femoral bowing. Computer-assisted surgery (CAS) and intra-articular bone resection with TKA are the treatments of choice for patients with ipsilateral extra-articular deformity. In theory, both CAS and intra-articular bone resection are beneficial in Ranawat type-II valgus arthritic knees with marked coronal femoral bowing deformity, but the literature on this topic is sparse. We compared the benefits of using these two techniques for TKA under this circumstance.

Methods: Patients who had Ranawat type-II valgus arthritic knees and marked coronal femoral bowing deformity and had undergone TKA at our hospital between 2005 and 2013 were enrolled in this retrospective study. Patients treated with CAS were assigned to the CAS-TKA group; patients treated with intra-articular bone resection were assigned to the Bone-Resect-TKA group. Radiographic parameters and clinical outcomes (International Knee Society (IKS) scores and patellar scores) in both groups were compared.

Results: Forty-seven patients (50 knees) met the inclusion criteria: 22 knees in the CAS-TKA group and 28 knees in the Bone-Resect-TKA group. Lateral retinaculum release was significantly $(P=0.008)$ higher in the Bone-Resect-TKA group. The joint-line was significantly properly restored in the CAS-TKA group $(P=0.011)$. The reconstructed mechanical axis was significantly $(P=0.012)$ closer to normal in the CAS-TKA group than in the Bone-Resect-TKA group. For component alignment, the femoral valgus and femoral flexion angles were significantly better in the CAS-TKA group ( $P=0.002$ and $P=0.006$, respectively), but not the tibial valgus, tibial flexion, or patellar tilting angles. IKS scores and patellar scores were not significantly different between groups at a mean follow-up of 60.2 months.

* Correspondence: b8601081@adm.cgmh.org.tw; wwh@cgmh.org.tw

${ }^{1}$ Chang Gung Memorial Hospital, Chiayi, Taiwan

Full list of author information is available at the end of the article 
(Continued from previous page)

Conclusions: CAS-TKA was effective for obtaining proper alignment and joint-line restoration in patients with Ranawat type-II valgus arthritic knees and marked coronal femoral bowing deformity, but not for yielding better clinical outcomes. Additional large-scale prospective randomized cohort studies with long-term follow-ups are necessary to make evidence-based recommendations.

Keywords: Bowing deformity of femur, Total knee arthroplasty, Genu valgus deformity, Computer-assisted surgery, Intra-articular bone resection

\section{Background}

Accurately restoring the mechanical axis (MA) of the limb, aligning components, and properly balancing soft tissue are vital for the long-term success of total knee arthroplasty (TKA) [1]. In most TKAs, alignments can be restored and soft tissue balanced using appropriate bone cuts and soft tissue releases. However, TKA becomes technically more challenging when an arthritic knee is associated with intra-articular and extra-articular deformities [2, 3].

TKA on Ranawat type-II valgus arthritic knees is a challenge for orthopedic surgeons because of its associated intra- and extra-articular bony abnormalities [4, 5]. When using a conventional system to guide alignment, the distortion of the bony canal and variations in femoral anatomy are likely to decrease accuracy and cause improper limb and component alignments [4-7]. Balancing the soft tissue in Ranawat type-II valgus arthritic knees is another challenge and might contribute to complications such as peroneal nerve palsy and patellar instability [6, 7]. Many surgeons find it difficult to correct a valgus deformity using a conventional alignment guiding system without also using a constrained implant $[4,5]$.

Marked coronal femoral bowing deformity is easily missed. Evidence of this deformity cannot be seen in a short knee film, and it does not present clinically or intraoperatively (Fig. 1) [8, 9]. A rather high prevalence has been reported in China, India, Japan, Korea, Singapore, Taiwan, and Turkey [9-11]. The marked coronal bowing deformity alters the relationship between the MA and anatomical axis (AA) of the femur, thereby affecting the postoperative MA and the placement of the femoral component [12-14].

Ranawat type-II valgus arthritic knee with marked coronal femoral bowing is an unusual and complex deformity. Associated intra- and extra-articular deformities, as well as a difficult sequential soft tissue balance, might decrease the incidence of satisfactory outcomes in patients with TKA. In patients with arthritic knee and ipsilateral extra-articular deformity, TKA using intra-articular bone resection $[15,16]$ and computer-assisted surgery (CAS) $[17,18]$ are reported effective for increasing the accuracy and reproducibility of limb and component alignment with fewer outliers. Theoretically, both CAS and intra-articular bone resection are beneficial in Ranawat type-II valgus arthritic knees with marked coronal femoral bowing deformity, but the literature on this topic is sparse. Thus, we hypothesized that CAS-TKA would be more efficacious than intra-articular bone resection-TKA, and we investigated their effect on the radiographic and clinical outcomes of TKA.

\section{Methods}

\section{Demographics}

All patients who underwent TKA at Chang Gung Memorial Hospital, Chiayi since 2002 were routinely enrolled in our arthroplasty registry. The cost of CAS is not reimbursed by Taiwan's National Health Insurance program, so after a complete explanation of the merits and disadvantages of both CAS-TKA and conventional techniques, the patients can choose the type of surgery they want. We prospectively collected clinical data: age, gender, diagnosis, length of hospital stay, surgical methods used, tourniquet time,

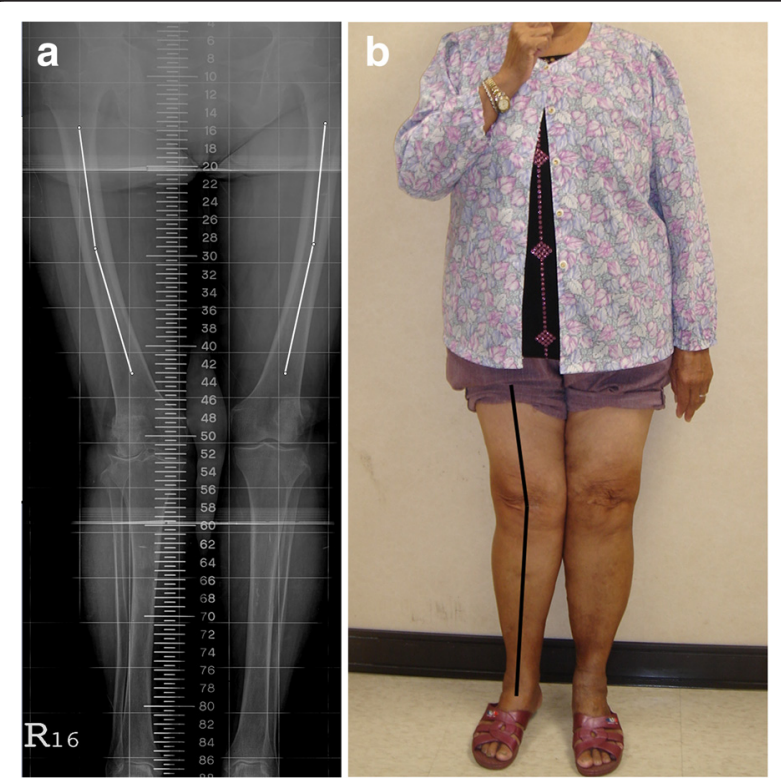

Fig. 1 A 79-year-old woman with right-knee osteoarthritis and a genu valgus deformity of the right lower limb. a This preoperative full-length standing scanogram of the lower extremity shows a marked bilateral coronal femoral bowing deformity (measured using the method described in Mullaji et al. [21]). b Marked coronal femoral bowing deformities do not present clinically 
total blood loss, complications associated with each surgical method, and preoperative and postoperative radiographic and clinical functional assessments.

Patient records in our arthroplasty database were manually reviewed to identify those patients with Ranawat type-II valgus arthritic knee and marked coronal femoral bowing who underwent a primary TKA. To minimize surgeon-related confounding factors, all of the TKAs we selected had been performed by the same surgeon (R.W.-W.H.), who has extensive experience using both conventional mechanical guides and computer-assisted navigation. To minimize implant-related confounding factors, the P.F.C. ${ }^{\bullet}$ Sigma ${ }^{\circ}$ Knee System (DePuy Synthes, Warsaw, IN, USA) was used with all TKAs. Those patients with (a) a minimum follow-up of $<24$ months, (b) an extra-articular deformity of the femur or tibia because of previous trauma or surgery, and (c) incomplete medical records, radiographic analyses, or clinical functional assessments were excluded.

The patients were then divided into two subgroups: the CAS-TKA group (treated with CAS-TKA) and the Bone-Resect-TKA group (treated with intra-articular bone resection).

\section{Assessments}

Preoperative and postoperative examinations of all patients included anteroposterior (AP) and lateral radiographs of the knee, a skyline view of the patellofemoral joint, and a full-length standing scanogram of the lower extremity [19]. These radiographs were used to measure the preoperative and postoperative MAs, valgus correction angles, and coronal femoral bowing angles. The magnitude of coronal femoral bowing was measured using the method previously described [8, 20]; femoral bowing greater than $5^{\circ}$ was considered substantial $[8,20]$ (Fig. 1). The lateral patellar tilt and displacement were measured using the method described in Laurin et al. [21, 22]. The postoperative patellar tilting angle was measured using the method previously described [23]. The position of the prosthetic joint line was measured using the method described in Figgie et al. [24] on radiographs taken before and after surgery. An adequately restored joint line was defined as a joint line within $5 \mathrm{~mm}$ of the normal position $[25,26]$. The component alignment angles-femoral valgus (FV) angle, tibial valgus (TV) angle, femoral flexion (FF) angle, and tibial flexion (TF) angle-were then measured [27]. The desired FV angle was based on the valgus correction angle of the distal femur, which was measured on the full-length standing scanogram of the lower extremity. The planned position was a TV angle of $90^{\circ}$ in the coronal plane and a FF angle of $0^{\circ}$ and TF angle of $87^{\circ}$ in the sagittal plane. The goal was to reconstruct the MA and component alignments to within $3^{\circ}$ of the proper position $[28,29]$. The radiographs were evaluated by an independent assessor blinded to the patients' demographic data. Intra-observer reliability, rated as "good" to "very good", was assessed using the method described previously [30].

Clinical outcomes were assessed using International Knee Society (IKS) scores [31] and the patellar score [32], which were checked preoperatively and at the last follow-up. The active maximum range of motion (ROM) of the knee was measured with a goniometer. All clinical outcomes were collected and analyzed by two independent surgeons who were blinded to the surgical techniques used, the group to which each patient had been assigned, and the patients' demographic data.

\section{Surgical technique}

All TKAs were done using an anterior midline longitudinal skin incision and a medial parapatellar arthrotomy. After osteophytes had been removed from the femur, the soft tissue of the lateral compartment was released from the proximal tibia. In the Bone-Resect-TKA group, an intramedullary (IM) alignment guidance system was used for femoral preparation and an extramedullary (EM) guidance system was used for tibial preparation. The intra-articular bone resection technique previously described $[15,16]$ was used. After the distal femoral and tibial resection had been done, the knee was placed in full extension and a spacer block was used to determine the extension gap, the appropriate thickness of the tibial insert, and the coronal alignment of the lower limb, and to validate the soft tissue balance. The anatomical landmarks were precisely identified for femoral chamfer cuts, and a surgical transepicondylar line was drawn to judge femoral rotation. Whiteside's line, the posterior condylar line, and the tibial cutting plane were supplemental in judging femoral rotation. After completing the osseous cuts of the femur, we placed the knee in $90^{\circ}$ of flexion and a spacer block was used to evaluate the flexion gap. The rotation of the tibial component was adjusted, making it parallel to the axis between the medial-third of the tibial tuberosity and the center of the tibial plateau. The posterior cruciate ligament (PCL) was evaluated using the pull-out lift-off test previously described [33]. The PCL was then recessed as needed from its insertion site in the tibia. The soft tissue balance was assessed in a trial reduction and achieved by sequentially releasing the tight structures, as recommended [34]. The tourniquet was then deflated, and the hemostasis and patellar tracking were assessed using the no-thumb test.

In the CAS-TKA group, the prostheses were implanted with the assistance of a computer tomography (CT)-free navigation system (BrainLAB, Munich, Germany). After the knee joint had been exposed, an adequate amount of synovium was removed to render registration precise. All anatomic landmarks were identified and then sequentially 
registered in the navigation system. The implant size and orientation were identified by dragging the pointer along the bone surface to reconstruct the three-dimensional bone model. Then, femoral preparation followed by tibial preparation was completed under the navigation. The femoral component was referenced parallel to the anterior cortex of the distal femur and the transepicondylar line. After completing the osseous cuts of the femur and tibia, PCL tension was assessed using the method previously described [33]. The soft tissue balance was assessed in a trial reduction, and proper balance was achieved by sequentially releasing the tight structures using real-time and quantitative feedback from the navigation [34]. The tourniquet was then deflated, hemostasis done, and patellar tracking assessed.

All patients enrolled in this study were treated with the same protocol, which included the use of wound suction drains for $48 \mathrm{~h}$ and a continuous passive motion machine for passive ROM exercises four times daily (30 min per exercise session) from the day of surgery until the day of discharge from the hospital. Under the supervision of a physical therapist, the patients started active knee-motion exercises and began standing at bedside or walking with crutches or a walker twice daily for 30-min sessions. All patients used crutches or a walker with full weight-bearing for 6 weeks and a cane when needed thereafter.

\section{Statistical analysis}

All data were collected and independently entered into a Microsoft Excel spreadsheet by two independent surgeons who were blinded to the surgical techniques used and the group to which each patient had been assigned. After the spreadsheets had been rechecked for missing and illogical data, the data were copied into SPSS 13.0 for Windows (SPSS Inc., Chicago, IL, USA) and analyzed. A $\chi^{2}$ test and Fisher's exact test were used to compare the quality of implantation (measured against the ideal position) between the two groups with these parameters. Student's $t$ test was used to compare the variables of age, body height, body weight, body mass index, hospital stay, tourniquet time, blood loss, difference in perioperative hemoglobin levels, follow-up time, functional results, and radiographic parameters. All data were analyzed by an independent statistician who was blinded to the surgical outcomes. Significance was set at $p<0.05$.

\section{Results}

\section{Demographic data}

Forty-seven patients (mean age 70 years; range 63 to 86 years) (50 knees 22 knees in the CAS-TKA group and 28 in the Bone-Resect-TKA group) were enrolled in this study. Degenerative osteoarthritis was diagnosed in all 50 knees. The mean body height was $156 \mathrm{~cm}$ (range
133 to $174 \mathrm{~cm}$ ), mean body weight $67 \mathrm{~kg}$ (range 35 to $95 \mathrm{~kg}$ ), and mean body mass index was $27.4 \mathrm{~kg} / \mathrm{m}^{2}$ (range 19.8 to $38.1 \mathrm{~kg} / \mathrm{m}^{2}$ ). The mean follow-up time was 60.2 months (range 24 to 97 months). There were no significant demographic differences between the two patient groups (Table 1).

\section{Perioperative data}

There was one significant perioperative difference between the two groups. To obtain adequate patellar tracking, 12 knees (42.9\%) in the Bone-Resect-TKA group, but only 2 knees $(9.1 \%)$ in the CAS-TKA group, required releasing the lateral retinaculum $(P=0.008)$. Total blood loss and perioperative hemoglobin levels were lower in the CAS-TKA group than those in the Bone-Resect-TKA group; however, the differences were not significant. There were no significant differences in the tourniquet time or the incidence of bone grafting (Table 2).

\section{Preoperative and postoperative lower limb alignment and} component alignment

The preoperative MA between the two groups was not significantly different. The postoperative MA was significantly $(P=0.012)$ closer to normal in the CAS-TKA group than that in the Bone-Resect-TKA group. The postoperative MA was corrected to $180^{\circ}$ (range 178 to $181^{\circ}$ ) in the former and $178^{\circ}$ (range 176 to $184^{\circ}$ ) in the latter (Table 2$)$. There was significantly $(P=0.011)$ less joint line elevation in the CAS-TKA group than that in the Bone-Resect-TKA group. For component alignment, the femoral valgus and femoral flexion angles were significantly $(P=0.002$ and $P=0.006$, respectively) better for the CAS-TKA group (Table 3). The percentage of procedures that had the ideal reconstructed MAs and component alignments was similar between the two groups (Table 4).

Table 1 Demographic data of the patients

\begin{tabular}{lccl}
\hline Parameters & CAS-TKA & Bone-Resect-TKA & $P$ \\
& $n=22$ & $n=28$ & value \\
\hline Age (years) & $70(63-86)$ & $71(64-85)$ & 0.895 \\
Body height $(\mathrm{cm})$ & $156(147-172)$ & $154(133-174)$ & 0.716 \\
Body weight $(\mathrm{kg})$ & $66(47-79)$ & $67(35-95)$ & 0.504 \\
Body mass index $\left(\mathrm{kg} / \mathrm{m}^{2}\right)$ & $26.8(21.8-31.1)$ & $27.1(19.8-38.1)$ & 0.324 \\
Hospital stay (days) & $6.6(5-10)$ & $6.3(5-10)$ & 0.114 \\
Follow-up time (months) & $61.5(24-82)$ & $59.5(26-97)$ & 0.148 \\
\hline
\end{tabular}

Data are mean (range). $P$ for between-group comparisons was determined using $t$ tests

CAS-TKA knees treated with computer-assisted surgery-total knee arthroplasty, Bone-Resect-TKA knees treated with intra-articular bone resection-total knee arthroplasty

Statistically significant $(P<0.05)$ 
Table 2 Perioperative data

\begin{tabular}{lccl}
\hline Parameters & $\begin{array}{l}\text { CAS-TKA } \\
n=22\end{array}$ & $\begin{array}{l}\text { Bone-Resect-TKA } \\
n=28\end{array}$ & $\begin{array}{l}P \\
\text { value }\end{array}$ \\
\hline $\begin{array}{l}\text { Perioperative data } \\
\text { Total blood loss (ml) }\end{array}$ & $601(215-770)$ & $762(285-895)$ & 0.071 \\
$\begin{array}{l}\text { Difference of perioperative } \\
\text { hemoglobin level (g/dL) }\end{array}$ & $1.1(0.4-2.1)$ & $1.8(0.6-2.5)$ & 0.112 \\
$\begin{array}{l}\text { Tourniquet time (minutes) } \\
\text { Lateral retinaculum for }\end{array}$ & $111(68-117)$ & $109(72-129)$ & 0.532 \\
patellar tracking & $2(9.1 \%)$ & $12(42.9 \%)$ & $0.008^{*}$ \\
Bone grafting & $10(45.5 \%)$ & $12(42.9 \%)$ & 0.540 \\
\hline
\end{tabular}

Data are mean (range) or (\%). $P$ for between-group comparisons was determined using $x^{2}$ tests for categorical variables and $t$ tests for continuous variables

CAS-TKA knees treated with computer-assisted surgery-total knee arthroplasty,

Bone-Resect-TKA knees treated with intra-articular bone resection-total knee arthroplasty

*Statistically significant $(p<0.05)$

\section{Functional results}

Clinically, the active ROM improved from $95^{\circ}$ to $115^{\circ}$ in the CAS-TKA group and from $99^{\circ}$ to $115^{\circ}$ in the BoneResect-TKA group. The mean patellar score improved postoperatively in both groups (from 16.3 to 26.9 in the CAS-TKA group and from 16.6 to 27.6 in the BoneResect-TKA group). On the IKS scoring system, the mean pain score improved from 16.3 to 46.7 in the CAS-TKA group and from 15.8 to 47.1 in the Bone-Resect-TKA

Table 3 Radiographic data

\begin{tabular}{|c|c|c|c|}
\hline Parameters & $\begin{array}{l}\text { CAS-TKA } \\
n=22\end{array}$ & $\begin{array}{l}\text { Bone-Resect-TKA } \\
n=28\end{array}$ & $\begin{array}{l}P \\
\text { value }\end{array}$ \\
\hline \multicolumn{4}{|l|}{ Radiographic data } \\
\hline Preoperative MA $\left(^{\circ}\right)$ & $193^{\circ}\left(192^{\circ}-198^{\circ}\right)$ & $194^{\circ}\left(191^{\circ}-196^{\circ}\right)$ & 0.954 \\
\hline Postoperative MA $\left(^{\circ}\right)$ & $180^{\circ}\left(178^{\circ}-181^{\circ}\right)$ & $178^{\circ}\left(176^{\circ}-184^{\circ}\right)$ & $0.012^{*}$ \\
\hline $\begin{array}{l}\text { Valgus correction angle } \\
\text { of the distal femur }\left({ }^{\circ}\right)\end{array}$ & $10^{\circ}\left(8^{\circ}-12^{\circ}\right)$ & $10^{\circ}\left(7^{\circ}-12^{\circ}\right)$ & 0.739 \\
\hline $\begin{array}{l}\text { Coronal femoral bowing } \\
\text { angle }\left(^{\circ}\right)\end{array}$ & $8^{\circ}\left(7^{\circ}-13^{\circ}\right)$ & $9^{\circ}\left(8^{\circ}-12^{\circ}\right)$ & 0.714 \\
\hline $\begin{array}{l}\text { Preoperative congruent } \\
\text { angle }\left({ }^{\circ}\right)\end{array}$ & $12^{\circ}\left(2^{\circ}-38^{\circ}\right)$ & $12^{\circ}\left(1^{\circ}-39^{\circ}\right)$ & 0.887 \\
\hline $\begin{array}{l}\text { Postoperative patellar } \\
\text { tilting angle }\left(^{\circ}\right)\end{array}$ & $2^{\circ}\left(1^{\circ}-4^{\circ}\right)$ & $3^{\circ}\left(2^{\circ}-5^{\circ}\right)$ & 0.855 \\
\hline Joint line elevation (mm) & $1(-1-3)$ & $3(2-6)$ & $0.011^{*}$ \\
\hline \multicolumn{4}{|l|}{ Component alignment } \\
\hline Femoral valgus angle $\left(^{\circ}\right)$ & $98^{\circ}\left(97^{\circ}-102^{\circ}\right)$ & $97^{\circ}\left(95^{\circ}-100^{\circ}\right)$ & $0.002^{*}$ \\
\hline Femoral flexion angle $\left(^{\circ}\right)$ & $1^{\circ}\left(0^{\circ}-7^{\circ}\right)$ & $3^{\circ}\left(0^{\circ}-7^{\circ}\right)$ & $0.006^{*}$ \\
\hline Tibial valgus angle $\left(^{\circ}\right)$ & $89^{\circ}\left(88^{\circ}-91^{\circ}\right)$ & $90^{\circ}\left(89^{\circ}-91^{\circ}\right)$ & 0.716 \\
\hline Tibial flexion angle $\left(^{\circ}\right)$ & $88^{\circ}\left(84^{\circ}-90^{\circ}\right)$ & $87^{\circ}\left(83^{\circ}-91^{\circ}\right)$ & 0.643 \\
\hline
\end{tabular}

Data are mean (range). $P$ for between-group comparisons was determined using $t$ tests

CAS-TKA knees treated with computer-assisted surgery-total knee arthroplasty, Bone-Resect-TKA knees treated with intra-articular bone resection-total knee arthroplasty, MA mechanical axis

*Statistically significant $(P<0.05)$
Table 4 Comparison of percentage of postoperative lower limb alignment (within $3^{\circ}$ deviation) and component alignment

\begin{tabular}{|c|c|c|c|}
\hline Parameters & $\begin{array}{l}\text { CAS-TKA } \\
n=22\end{array}$ & $\begin{array}{l}\text { Bone-Resect-TKA } \\
n=28\end{array}$ & $\begin{array}{l}P \\
\text { value }\end{array}$ \\
\hline \multicolumn{4}{|c|}{ Mechanical axis within $3^{\circ}$ deviation } \\
\hline & $20(90.9 \%)$ & $18(64.3 \%)$ & $0.029^{*}$ \\
\hline \multicolumn{4}{|l|}{ Component positioning } \\
\hline Femoral valgus angle & $20(90.9 \%)$ & $18(64.3 \%)$ & $0.029^{*}$ \\
\hline Femoral flexion angle & 19 (86.4 \%) & $17(60.7 \%)$ & $0.044^{*}$ \\
\hline Tibial valgus angle & $22(100 \%)$ & $26(92.9 \%)$ & 0.309 \\
\hline Tibial flexion angle & 20 (90.9 \%) & $25(89.3 \%)$ & 0.616 \\
\hline \multicolumn{4}{|c|}{$\begin{array}{l}\text { Data are } \mathrm{n}(\%) . P \text { for between-group comparisons was determined using } X^{2} \text { tests } \\
\text { CAS-TKA knees treated with computer-assisted surgery-total knee arthroplasty, } \\
\text { Bone-Resect-TKA knees treated with intra-articular bone resection-total } \\
\text { knee arthroplasty } \\
{ }^{*} \text { Statistically significant }(P<0.05)\end{array}$} \\
\hline
\end{tabular}

group; the mean clinical knee score improved from 39.5 to 96.2 and from 39.1 to 97.6 , respectively; and the mean functional knee score improved from 35.2 to 95.4 and from 34.3 to 96.6 , respectively. Although there were marked improvements in all of the postoperative scores, there were no significant preoperative or postoperative differences between the two groups (Table 5).

Table 5 Preoperative and postoperative patellar, IKS, and ROM functional scores

\begin{tabular}{|c|c|c|c|}
\hline Parameters & $\begin{array}{l}\text { CAS-TKA } \\
n=22\end{array}$ & $\begin{array}{l}\text { Bone-Resect-TKA } \\
n=28\end{array}$ & $\begin{array}{l}P \\
\text { value }\end{array}$ \\
\hline \multicolumn{4}{|l|}{ Preoperative functional score } \\
\hline Patellar score (points) & $16.3(10-24)$ & $16.6(10-24)$ & 0.583 \\
\hline IKS pain score (points) & $16.3(10-20)$ & $15.8(10-20)$ & 0.335 \\
\hline $\begin{array}{l}\text { IKS clinical knee score } \\
\text { (points) }\end{array}$ & $39.5(11-65)$ & $39.1(16-60)$ & 0.714 \\
\hline $\begin{array}{l}\text { IKS functional knee score } \\
\text { (points) }\end{array}$ & $35.2(20-55)$ & $34.3(20-50)$ & 0.504 \\
\hline Active range of motion $\left(^{\circ}\right)$ & $95^{\circ}\left(85^{\circ}-120^{\circ}\right)$ & $99^{\circ}\left(90^{\circ}-120^{\circ}\right)$ & 0.960 \\
\hline \multicolumn{4}{|l|}{ Postoperative functional score } \\
\hline Patellar score (points) & $26.9(20-30)$ & $27.6(21-30)$ & 0.822 \\
\hline IKS pain score (points) & $46.7(40-50)$ & $47.1(40-50)$ & 0.716 \\
\hline $\begin{array}{l}\text { IKS clinical knee score } \\
\text { (points) }\end{array}$ & $96.2(87-100)$ & $97.6(90-100)$ & 0.887 \\
\hline $\begin{array}{l}\text { IKS functional knee score } \\
\text { (points) }\end{array}$ & $95.4(80-100)$ & $96.6(90-100)$ & 0.668 \\
\hline Active range of motion $\left(^{\circ}\right)$ & $115^{\circ}\left(100^{\circ}-25^{\circ}\right)$ & $115^{\circ}\left(105^{\circ}-125^{\circ}\right)$ & 0.541 \\
\hline
\end{tabular}

Data are mean (range). $P$ for between-group comparisons was determined using $t$ tests

CAS-TKA knees treated with computer-assisted surgery-total knee arthroplasty, Bone-Resect-TKA knees treated with intra-articular bone resection-total knee arthroplasty, IKS Score International Knee Society Score Statistically significant $(P<0.05)$ 


\section{Complications}

There were no complications in any of the 50 knees. Specifically, no patient required advancement of the medial collateral ligament (MCL) or was converted to a posterior-stabilized prosthesis or constrained components because of an excessively bony resection or inadequate soft tissue release during the operation. There were no cases of wound infection, peroneal nerve neurapraxia, pulmonary emboli, or deep vein thrombosis. No periprosthetic fractures, joint instability, or patellar tracking problems were encountered. No patients showed loosening or osteolysis on radiography at the time of the last follow-up, and no patients underwent revision surgery for any reason.

\section{Discussion}

The most important finding of this study was that CAS was more efficacious than intra-articular resection for facilitating a properly reconstructed MA, femoral component placement, and restoration of the joint-line when performing TKA on a patient with a Ranawat type-II valgus arthritic knee with a marked coronal femoral bowing deformity. However, CAS did not yield a better clinical outcome at a mean follow-up of 60.2 months.

The long-term outcome of TKA depends on good component positioning and a reconstructed MA that is within $3^{\circ}$ of neutral in the coronal plane [1-3]. However, TKA on either Ranawat type-II valgus arthritic knees or on knees with marked coronal femoral bowing deformities is technically demanding because associated intra-articular bony abnormalities (e.g., distal femoral hypoplasia, posterior femoral condylar erosion, and patellar maltracking) and extra-articular bony abnormalities (e.g., increased femoral neck-shaft angle and metaphyseal remodeling of both the femur and the tibia) might render the use of conventional guidance systems inadvisable $[6,7]$. However, from an anatomical perspective, the angular relationship between the MA and AA of the femur is influenced by a marked bowing deformity in the coronal plane [8-11]. This anatomic feature is easily overlooked because it is neither clinically apparent nor evident on short-film radiographs of the knee [19] (Fig. 1).

When using conventional TKA, the choice of cutting block used for a distal femoral bone cut perpendicular to the MA of the femur depends upon the valgus correction angle of the distal femur. For valgus arthritic knees, Ranawat et al. [5] suggested using a $3^{\circ}$ valgus distal femoral cutting block rather than the usual $5^{\circ}$ to $7^{\circ}$ cutting block. However, we found that the femoral bowing in patients with Ranawat type-II valgus arthritic knees with marked coronal femoral bowing deformities resulted in a more variable valgus correction angle of the distal femur (range $7^{\circ}$ to $12^{\circ}$ ). Routine use of a $3^{\circ}$ valgus distal femoral cut might not be advisable. Any femoral cutting system must incorporate appropriate preoperative planning to adjust the cuts to accommodate the deformity. However, the majority of currently available femoral jigs do not provide a broad enough choice of valgus cut angles to realize an ideal reconstructed MA in patients with such deformities [12-14].

Staged and simultaneous corrective osteotomy and TKA have been advocated to achieve proper limb alignment and better ligament balancing when a patient has an arthritic knee with an extra-articular deformity [35-37]. However, this technique might be associated with substantial complications, including delayed union or nonunion at the osteotomy site, failure of internal fixation, infection of the osteotomy site, and arthrofibrosis [15]. Hadjicostas et al. [38] studied 15 patients with valgus deformities of $17^{\circ}$ to $27^{\circ}$ and reported excellent mid-term results using CAS to guide a simultaneous osteotomy of the lateral femoral condyle before TKA. The deformities in their patients were much larger in the coronal plane than were the deformities in our series (range $11^{\circ}$ to $18^{\circ}$ ) and were, therefore, difficult to correct with intra-articular bone resection or CAS. In addition, if a deformity in the supracondylar area is $\geq 20^{\circ}$, soft tissue balancing will be difficult after joint line resection. Therefore, staged or simultaneous corrective osteotomy and TKA should be considered in patients with a large deformity [15]. In this study, we used a previously described intra-articular bone resection method $[15,16]$. Use of an IM guidance system with a modified starting hole in the knee has been reported to be effective for arthritis of the knee with ipsilateral extraarticular deformity [15]. However, this method also increases the risk of improper postoperative MA and femoral component malalignment. The most likely reason is that the incomplete insertion of IM rods may result in a subsequent erroneous distal femur resection [3]. On the other hand, with CAS-TKA, the surgeon can focus on the centers of the hip, knee, and ankle joints and ignore any extra-articular deformities of the femur and tibia, which allows for cuts that can provide the desired reconstructed alignment of the limb and its components [9, 13, 14] (Fig. 2).

Malrotation of the femoral component affects patellofemoral tracking and might increase contact pressure, which leads to accelerated and excessive wear on the patellar button [39]. In Ranawat type-II valgus arthritic knees, the lateral femoral condyle deficiencies often render the posterior condylar axis inaccurate as a reference for determining femoral component rotation. A fixed $3^{\circ}$ external rotation might not be suitable under this circumstance [4-7]. In the current study, we used the transepicondylar axis as a reference to judge femoral rotation. Whiteside's line, the posterior condylar line, and the tibial cutting plane were supplemental in judging femoral rotation. However, significantly more knees in the Bone-Resect- 


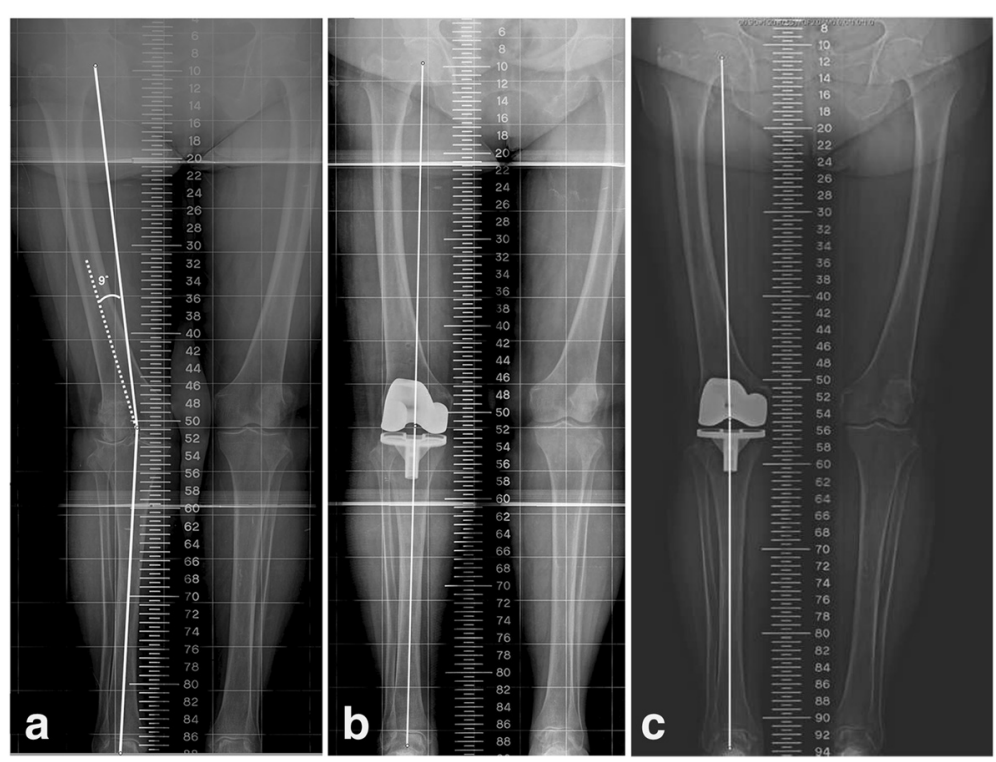

Fig. 2 Representative results with one patient who had undergone CAS-TKA for Ranawat type-ll valgus arthritic knees and marked coronal femoral bowing. a This preoperative full-length standing scanogram of the lower extremity shows Ranawat type-II valgus arthritic knees with a mechanical anatomical axis of 193 and a $9^{\circ}$ femoral valgus resection angle. $\mathbf{b}$ A postoperative radiograph showing complete restoration of limb alignment after undergoing CAS-TKA. c Eight years later, the limb remains in excellent alignment

TKA group $(12=42.9 \%)$ than in the CAS-TKA group $(2=9.1 \%)$ required release of the lateral retinaculum to obtain adequate patellar tracking. When using CAS-TKA, the surgeon can judge the accuracy of the cutting jig in the axial plane using real-time and quantitative feedback from the navigation. A mistake in visual judgment of a chamfer block in the axial plane might be the confounding factor that results in inadequate femoral rotation alignment in conventional TKA.

Balancing the soft tissue in Ranawat type-II valgus arthritic knees is another challenge [4, 5]. A precise osseous cutting technique can prevent a large extension gap and lax MCL. However, Ranawat type-II valgus arthritic knees have more medial soft tissue stretching and lateral soft tissue contraction, which might contribute to inappropriate soft tissue management. Over-releasing of soft tissue might result in component malalignment and an unplanned conversion to a constrained prosthesis [3-7]. Thick polyethylene tibial inserts have been used to compensate for excessive laxity, but this risks elevating the joint line and increasing the TKA failure rate [40]. Joint line elevation secondary to placing a thick polyethylene spacer increases the risk of peroneal nerve neuropraxia, and patellofemoral contact forces might contribute to postoperative complications such as pain, polyethylene wear, and inferior clinical results [41]. Limiting joint line elevation to $<5 \mathrm{~mm}$ in primary TKAs is considered satisfactory because it does not affect clinical outcomes [26]. To overcome these difficulties, many soft tissue handling procedures have been used with consistent and satisfactory results [6]. However, the ideal soft tissue balance still largely depends upon the surgeon's experience. Lack of a quantitative and objective monitoring system might reduce the incidence of satisfactory TKA outcomes in the hands of less experienced surgeons. With the real-time and quantitative feedback of a navigation system, surgeons can provide a proper soft tissue balance and prevent joint-line elevation. Ensini et al. [42] and Chou et al. [43] reported that the joint line is well restored and the risk of overstuffing is limited with CAS-TKA. We too found less joint line elevation with CAS-TKA than with conventional TKA.

This study has some limitations. First, it has all the inherent limitations and biases of a retrospective study. However, all patients were treated by the same experienced surgeon using the same protocol, which decreases the effects of some confounding factors. Second, this was a radiographic and short-term clinical follow-up study. Although the CAS-TKA group had higher percentages of ideal postoperative MAs and component alignment, CASTKA did not yield better clinical results than did BoneResect-TKA at a mean follow-up of 60.2 months. One of the reasons for not finding significant differences in clinical outcomes is the duration of follow-up. If the minimum follow-up period is more than 10 years, differences in failure rates between well-aligned and malaligned TKAs might become apparent. Additional long-term studies on CAS-TKA are needed to determine whether radiographic benefits result in better long-term clinical outcomes. Third, there were only 50 knees in this study, which 
reflects the relative rarity of Ranawat type-II valgus arthritic knees with marked coronal femoral bowing deformities in patients undergoing TKA. One study [44], using the IKS scoring system to calculate a sample size with a power of $80 \%$ and a significance of 0.05 to detect a difference of 5 points in the IKS score (estimated SD of 8), found that 33 knees were required per group. With only 22 cases of CAS-TKA, our study was too underpowered to show significant differences with Bone-Resect-TKA. Although it is always better to have more patients in a prospective study to clarify the effect of CAS-TKA on proper alignment and clinical outcomes, it would be difficult to do a prospective randomized large-scale cohort study based on this rare deformity. Finally, the current study did not include CT-based navigation, which might have an accuracy advantage in rotational alignment because it allows accurate preoperative planning on patient-specific threedimensional bone models. However, CT-based navigation generates more radiation and requires additional time for preoperative planning, and the scanning increases costs. After the anatomic landmarks have been adequately identified, image-free navigation might be sufficient for proper component alignment.

\section{Conclusions}

Ranawat type-II valgus arthritic knee with marked coronal femoral bowing reduces the accuracy of restoring postoperative MA, femoral component alignment, and the jointline elevation level when intra-articular resection is used. Instead of the traditional fixed valgus correction angle of the distal femur in Asian patients, the angle first needs to be determined and then adjusted using a full-length standing scanogram of the lower extremity. Modifying the femoral jig to provide a wider choice of valgus cut angles or using a staged or simultaneous femoral osteotomy combined with TKA are all viable options. Our data suggest that CAS-TKA can be an effective alternative for restoring the joint line, properly aligning the limb, and accurately positioning the components. However, we found no significant difference in clinical outcomes between the CAS-TKA and Bone-Resect-TKA groups. Studies with long-term follow-ups are needed to determine whether the improvement in radiographic results actually translates to better clinical outcomes.

\section{Abbreviations}

AA, anatomical axis; CAS-TKA, computer-assisted surgery total knee arthroplasty; $\mathrm{EM}$, extramedullary; $F \mathrm{~F}$, femoral flexion angle; $\mathrm{FV}$, femoral valgus angle; IKS, International Knee Society; IM, intramedullary; MA, mechanical axis; ROM, range of motion; TV, tibial valgus angle; TF, tibial flexion angle; TKA, total knee arthroplasty

\section{Acknowledgements}

We thank Ms. Yu-Shuan Lin for recording radiographic and functional data and for assisting with the statistical analyses.
Funding

There was no external funding for this study.

\section{Availability of data and materials}

The dataset supporting the conclusions of this article is included within the article. Data are available from the Chang Gung Memorial Hospital Data Access/Ethics Committee for researchers who meet the criteria for access to confidential data.

\section{Authors' contributions}

T-WH, MSL, RW-WH, and K-CH contributed substantially to the conception and design of the study. C-YL, S-JL, S-HS, and Y-HT analyzed and interpreted the data. T-WH and P-YC drafted the article and revised it critically for important intellectual content. All authors reviewed and edited the manuscript and approved the final version of the manuscript.

\section{Competing interests}

The authors declare that they have no competing interests.

\section{Consent for publication}

Consents regarding individual details, images, and videos were obtained from all patients.

\section{Ethics approval and consent to participate}

This retrospective study was approved by the Ethics Committee and Institutional Review Board of Chang Gung Memorial Hospital (99-2025B), and all patients provided signed informed consent.

\section{Author details}

${ }^{1}$ Chang Gung Memorial Hospital, Chiayi, Taiwan. ${ }^{2}$ Kaohsiung Chang Gung Memorial Hospital, Kaohsiung, Taiwan. ${ }^{3}$ Chang Gung University, Taoyuan, Taiwan.

Received: 27 March 2016 Accepted: 15 July 2016

Published online: 03 August 2016

\section{References}

1. Huang TW, Hsu WH, Peng KT, Hsu RW, Weng YJ, Shen WJ. Total knee arthroplasty with use of computer-assisted navigation compared with conventional guiding systems in the same patient: radiographic results in Asian patients. J Bone Joint Surg Am. 2011;93:1197-202.

2. Lin SJ, Lee CY, Huang KC, Peng KT, Huang TW, Lee MS, Hsu RW, Shen WJ. Improved femoral component rotation in advanced genu valgum deformity using computer-assisted measured resection total knee arthroplasty. J Orthop Surg Res. 2015;10:135-43.

3. Mullaji AB, Shetty GM, Lingaraju AP, Bhayde S. Which factors increase risk of malalignment of the hip-knee-ankle axis in TKA? Clin Orthop Relat Res. 2013;471:134-41.

4. Elkus M, Ranawat CS, Rasquinha VJ, Babhulkar S, Rossi R, Ranawat AS. Total knee arthroplasty for severe valgus deformity. Five to fourteen-year follow-up. J Bone Joint Surg Am. 2004;86:2671-6.

5. Ranawat AS, Ranawat CS, Elkus M, Rasquinha VJ, Rossi R, Babhulkar S. Total knee arthroplasty for severe valgus deformity. J Bone Joint Surg Am. 2005;87:271-84.

6. Huang TW, Lee CY, Lin SJ, Peng KT, Huang KC, Lee MS, Hsu RW, Shen WJ. Comparison of computer-navigated and conventional total knee arthroplasty in patients with Ranawat type-II valgus deformity: medium-term clinical and radiological results. BMC Musculoskelet Disord. 2014;15:390-8.

7. Huang TW, Kuo LT, Peng KT, Lee MS, Hsu RW. Computed tomography evaluation in total knee arthroplasty: computer-assisted navigation versus conventional instrumentation in patients with advanced valgus arthritic knees. J Arthroplasty. 2014;29:2363-8.

8. Mullaji A, Shetty GM. Computer-assisted total knee arthroplasty for arthritis with extra-articular deformity. J Arthroplasty. 2009;24:1164-1169.e1.

9. Huang TW, Hsu WH, Peng KT, Hsu RW. Total knee replacement in patients with significant femoral bowing in the coronal plane: a comparison of conventional and computer-assisted surgery in an Asian population. J Bone Joint Surg (Br). 2011;93:345-50.

10. Soh HH, Chua IT, Kwek EB. Atypical fractures of the femur: effect of anterolateral bowing of the femur on fracture location. Arch Orthop Trauma Surg. 2015;135:1485-90. 
11. Schilcher J, Howe TS, Png MA, Aspenberg P, Koh JS. Atypical fractures are mainly subtrochanteric in Singapore and diaphyseal in Sweden: a cross-sectional study. J Bone Miner Res. 2015;30:2127-32.

12. Huang TW, Peng KT, Huang KC, Lee MS, Hsu RW. Differences in component and limb alignment between computer-assisted and conventional surgery total knee arthroplasty. Knee Surg Sports Traumatol Arthrosc. 2014;22:2954-61.

13. Lee CY, Lin SJ, Kuo LT, Peng KT, Huang KC, Huang TW, Lee MS, Hsu RW, Shen WJ. The benefits of computer-assisted total knee arthroplasty on coronal alignment with marked femoral bowing in Asian patients. J Orthop Surg Res. 2014;9:122-31.

14. Huang TW, Lee CY, Lin SJ, Lee MS, Hsu RW, Shen WJ. The influence of alignment on midterm outcome after total knee arthroplasty in patients with marked coronal femoral bowing. J Arthroplasty. 2015;30:1531-6.

15. Wang JW, Wang CJ. Total knee arthroplasty for arthritis of the knee with extra-articular deformity. J Bone Joint Surg Am. 2002;84:1769-74.

16. Wang JW, Chen WS, Lin PC, Hsu CS, Wang CJ. Total knee replacement with intra-articular resection of bone after malunion of a femoral fracture: can sagittal angulation be corrected? J Bone Joint Surg (Br). 2010;92:1392-6.

17. Chou WY, Ko JY, Wang CJ, Wang FS, Wu RW, Wong T. Navigation-assisted total knee arthroplasty for a knee with malunion of the distal femur. J Arthroplasty. 2008;23:1239.e13-19.

18. Bottros J, Klika AK, Lee HH, Polousky J, Barsoum WK. The use of navigation in total knee arthroplasty for patients with extra-articular deformity. J Arthroplasty. 2008;23:74-8.

19. Hsu RW, Himeno S, Coventry MB, Chao EY. Normal axial alignment of the lower extremity and load-bearing distribution at the knee. Clin Orthop Relat Res. 1990;255:215-27.

20. Mullaji AB, Marawar SV, Mittal V. A comparison of coronal plane axial femoral relationships in Asian patients with varus osteoarthritic knees and healthy knees. J Arthroplasty. 2009;24:861-7.

21. Laurin CA, Levesque HP, Dussault R, Labelle H, Peides JP. The abnormal lateral patellofemoral angle. J Bone Joint Surg Am. 1978;60:55-60.

22. Laurin CA, Dussault $R$, Levesque HP. The tangential X-ray investigation of the patellofemoral joint: $X$-ray technique, diagnostic criteria and their interpretation. Clin Orthop Relat Res. 1979;144:16-26.

23. Kawahara S, Okazaki K, Matsuda S, Nakahara H, Okamoto S, Iwamoto Y. Internal rotation of femoral component affects functional activities after TKA—survey with the 2011 Knee Society Score. J Arthroplasty. 2014;29:2319-23.

24. Figgie 3rd HE, Goldberg VM, Heiple KG, Moller 3rd HS, Gordon NH. The influence of tibial-patellofemoral location on function of the knee in patients with the posterior stabilized condylar knee prosthesis. J Bone Joint Surg Am. 1986;68:1035.

25. Yang JH, Seo JG, Moon YW, Kim MH. Joint line changes after navigation-assisted mobile-bearing TKA. Orthopedics. 2009;32:35-9.

26. Porteous AJ, Hassaballa MA, Newman JH. Does the joint line matter in revision total knee replacement. J Bone Joint Surg (Br). 2008;90:879-84.

27. Ewald FC. The Knee Society total knee arthroplasty roentgenographic evaluation and scoring system. Clin Orthop Relat Res. 1989;248:9-12.

28. Kim YH, Kim JS, Choi Y, Kwon OR. Computer-assisted surgical navigation does not improve the alignment and orientation of the components in total knee arthroplasty. J Bone Joint Surg Am. 2009;91:14-9.

29. Kim YH, Park JW, Kim JS, Park SD. The relationship between the survival of total knee arthroplasty and postoperative coronal, sagittal and rotational alignment of knee prosthesis. Int Orthop. 2014;38:379-85.

30. Konigsberg B, Hess R, Hartman C, Smith L, Garvin KL. Inter- and intraobserver reliability of two-dimensional CT scan for total knee arthroplasty component malrotation. Clin Orthop Relat Res. 2014;472:212-7.

31. Insall JN, Dorr LD, Scott RD, Scott WN. Rationale of the Knee Society clinical rating system. Clin Orthop Relat Res. 1989;248:13-4.

32. Feller JA, Bartlett RJ, Lang DM. Patellar resurfacing versus retention in total knee arthroplasty. J Bone Joint Surg (Br). 1996;78:226-8.

33. Scott RD, Chmell MJ. Balancing the posterior cruciate ligament during cruciateretaining fixed and mobile-bearing total knee arthroplasty: description of the pull-out lift-off and slide-back tests. J Arthroplasty. 2008;23:605-8.

34. Whiteside LA. Correction of ligament and bone defects in total arthroplasty of the severely valgus knee. Clin Orthop Relat Res. 1993;288:234-45.

35. Yagi K, Matsui Y, Nakano S, Egawa H, Tsutsui T, Kawasaki Y, Takata S, Yasui N. Treatment of knee osteoarthritis associated with extra-articular varus deformity of the femur: staged total knee arthroplasty following corrective osteotomy. J Orthopaedic Sci. 2006;11:386-9.
36. Lonner JH, Siliski JM, Lotke PA. Simultaneous femoral osteotomy and total knee arthroplasty for treatment of osteoarthritis associated with severe extra-articular deformity. J Bone Joint Surg Am. 2000;82:342-8.

37. Moyad TF, Estok D. Simultaneous femoral and tibial osteotomies during total knee arthroplasty for severe extra-articular deformity. J Knee Surg. 2009;22:21-6.

38. Hadjicostas PT, Soucacos PN, Thielemann FW. Computer-assisted osteotomy of the lateral femoral condyle with non-constrained total knee replacement in severe valgus knees. J Bone Joint Surg (Br). 2008;90:1441-5.

39. Verlinden C, Uvin P, Labey L, Luyckx JP, Bellemans J, Vandenneucker H. The influence of malrotation of the femoral component in total knee replacement on the mechanics of patellofemoral contact during gait: an in vitro biomechanical study. J Bone Joint Surg (Br). 2010;92:737-42.

40. Berend ME, Davis PJ, Ritter MA, Keating EM, Faris PM, Meding JB, Malinzak RA. "Thicker" polyethylene bearings are associated with higher failure rates in primary total knee arthroplasty. J Arthroplasty. 2010;25 suppl 6:17-20.

41. Konig C, Sharenkov A, Matziolis G, Taylor WR, Perka C, Duda GN, Heller MO. Joint line elevation in revision TKA leads to increased patellofemoral contact forces. J Orthop Res. 2010;28:1-5.

42. Ensini A, Catani F, Biasca N, Belvedere C, Giannini S, Leardini A. Joint line is well restored when navigation surgery is performed for total knee arthroplasty. Knee Surg Sports Traumatol Arthrosc. 2012;20:495-502.

43. Chou WY, Siu KK, Ko JY, Chen JM, Wang CJ, Wang FS, Wong T. Preoperative templating and computer-assisted total knee arthroplasty for arthritic valgus knee. J Arthroplasty. 2013;28:1781-7.

44. Lizaur-Utrilla A, Sanz-Reig J, Trigueros-Rentero MA. Greater satisfaction in older patients with a mobile-bearing compared with fixed-bearing total knee arthroplasty. J Arthroplasty. 2012;27:207-12.

\section{Submit your next manuscript to BioMed Central and we will help you at every step:}

- We accept pre-submission inquiries

- Our selector tool helps you to find the most relevant journal

- We provide round the clock customer support

- Convenient online submission

- Thorough peer review

- Inclusion in PubMed and all major indexing services

- Maximum visibility for your research

Submit your manuscript at www.biomedcentral.com/submit
) Biomed Central 